\title{
Oxigenoterapia Hiperbárica Enquanto Terapêutica Complementar da Úlcera do Pé Diabético: Útil ou Inútil? - Uma Revisão da Literatura
}

\section{Hyperbaric Oxygen Therapy as a Complementary Therapy for Diabetic Foot Ulcer: Useful or Useless? - A Literature Review}

Rodrigo Vicente ${ }^{1}$, Diogo Alpuim Costa², Miguel Castelo-Branco ${ }^{3}$

Autor Correspondente/Corresponding Author:

Rodrigo Vicente [rodrigovicente92@gmail.com]

Rua Alves Gouveia, n5 $1^{\circ}$ esq., 1800-021 Lisboa, Portugal

ORCID iD: 0000-0001-6780-4271

\section{RESUMO}

INTRODUÇÃO: O pé diabético é uma complicação frequente da diabetes mellitus com importante repercussão a nível clínico, psicossocial e económico, exigindo dos prestadores de cuidados de saúde uma abordagem terapêutica multimodal. Definiu-se como objetivo principal a revisão da literatura sobre o tratamento complementar do pé diabético com oxigenoterapia hiperbárica e as suas perspetivas futuras no tratamento desta complicação.

MATERIAL E MÉTODOS: Pesquisa bibliográfica através da PubMed, CINAHL, Cochrane e Google Académico desde 2014, utilizando os seguintes descritores: pé diabético, úlcera diabética e oxigenoterapia hiperbárica. Foram analisadas trinta e duas publicações, tendo sido selecionados seis artigos de revisão.

RESULTADOS: A oxigenoterapia hiperbárica parece eficaz na cicatrização aos seis meses, promissora na cicatrização de lesões com mais de doze meses de evolução e associada a uma baixa incidência de efeitos adversos. A saturação de oxigénio transcutânea foi maior e houve menor incidência de amputação major dos membros inferiores no subgrupo de doentes tratados com esta terapia.

DISCUSSÃO: A evidência atual aponta para um benefício clínico da oxigenoterapia hiperbárica enquanto modalidade terapêutica complementar do pé diabético. Ainda não é totalmente conhecido o perfil clínico ideal do doente que beneficiará deste tipo de tratamento pelas heterogeneidades e limitações metodológicas dos estudos considerados. É importante a realização de mais ensaios clínicos prospetivos, aleatorizados e controlados que consubstanciem a sua eficácia.

PALAVRAS-CHAVE: Oxigenoterapia Hiperbárica; Pé Diabético/tratamento

1. Serviço de Oncologia Médica, Hospital Professor Doutor Fernando Fonseca, Amadora, Portugal. 2. Centro de Medicina Subaquática e Hiperbárica, Lisboa, Portugal; Departamento de Hemato-Oncologia, CUF Instituto de Oncologia, Lisboa, Portugal. 3.Faculdade de Ciências da Saúde da Universidade da Beira Interior, Covilhã, Portugal. 


\begin{abstract}
INTRODUCTION: Diabetic foot is a frequent complication of diabetes mellitus with relevant economic and psychosocial issues, which requires optimisation of the therapeutic approach from health care providers. The main purpose was to review the literature on the clinical utility and future perspectives of hyperbaric oxygen therapy in the adjunctive treatment of the diabetic foot.
\end{abstract}

MATERIAL AND METHODS: PubMed, CINAHL, Cochrane and Google Academic search engines were used, for articles since 2014, with an analysis of the following keywords: diabetic foot, diabetic ulcer and hyperbaric oxygen therapy. Thirty-two publications were analysed and six review articles selected.

RESULTS: Hyperbaric oxygen has been reported to benefit healing process in follow-up periods up to six months and promising for a follow-up equal to or greater than twelve months. Low incidence of side effects was reported. The transcutaneous oxygen saturation of the foot was more significant in the groups of patients treated with this complementary therapy, and there was a decrease in the need for major amputation of the lower limbs.

DISCUSSION: Current evidence points to the clinical utility of hyperbaric oxygen therapy as a complementary treatment. It has yet to be found the optimal clinical profile that would benefit from this type of treatment due to limitations on the analysed studies. Randomised, prospective clinical trials are essential to confirm these findings.

KEYWORDS: Diabetic Foot/therapy; Hyperbaric Oxygenation

\section{INTRODUÇÃO}

Dados da Organização Mundial de Saúde (OMS) revelam a tendência para o aumento da prevalência de diabetes mellitus (DM) na população mundial com idade superior a 18 anos. Em 2015, estimou-se a existência de 415 milhões de adultos diabéticos no mundo. ${ }^{1}$ Em Portugal registou-se na faixa etária entre os 20 e 79 anos uma prevalência de 13,3\%, uma das mais altas da Europa. ${ }^{2,3}$ Em 2040, as estimativas a nível mundial apontam para que o número de casos diabéticos reportados ascenda aos 642 milhões. ${ }^{3}$

A úlcera do pé tem uma prevalência global de 6,3\% entre diabéticos, ${ }^{4}$ constituindo-se como uma condição neuropática facilitada por traumatismo prévio, que pode ou não estar associada a doença vascular periférica. Estas lesões são agravadas frequentemente por infeções e/ ou gangrena. ${ }^{5}$ A amputação, com um risco de 15 vezes superior na população com diagnóstico de DM, é o desfecho mais temido, mas potencialmente prevenível. 6,7 Cerca de $80 \%$ dos casos de amputação da extremidade distal inferior reportados em doentes diabéticos são precedidos por úlcera. ${ }^{8}$

No âmbito do Programa Nacional de Prevenção e Controlo da Diabetes, a circular normativa de 2010 N5/ PNPCD regulamenta os procedimentos a adotar pelos serviços e prestadores de cuidados de saúde para uma abordagem sistematizada do pé diabético. ${ }^{9}$ Não obstante, após um ano de tratamento standard, a taxa de cicatrização completa alcança valores que rondam os 60\%.10
A prestação de cuidados contínuos às úlceras é um procedimento dispendioso e moroso, com uma taxa de recorrência de $70 \%$ das feridas num período de cinco anos. ${ }^{11} \mathrm{O}$ estudo EURODIALE 2016, realizado pelo European Study Group on Diabetes and The Lower Extremity, revelou que o tratamento de uma úlcera não infetada envolve um custo médio de $10000 €$, aumentando para os 17 000€ em caso de infeção e doença arterial periférica concomitante. ${ }^{10}$

O pé diabético refratário ao tratamento convencional e os seus elevados custos económicos associados, em paralelo com a investigação crescente na área, permitiram o aparecimento e desenvolvimento de outros tratamentos para este contexto clínico. Neste âmbito, a oxigenoterapia hiperbárica tem vindo a constituir-se como uma modalidade terapêutica complementar em centros certificados e especializados. ${ }^{12-14}$

A oxigenoterapia hiperbárica (OHB) é um tratamento baseado na inalação de $\mathrm{O}_{2}$ puro (100\%) num ambiente sob uma pressão atmosférica superior à existente ao nível da superfície do mar (1 atmosfera absoluta - ATA). As sessões de $\mathrm{OHB}$ são realizadas no interior de câmaras hiperbáricas que constituem compartimentos estanques, sendo classificadas como dispositivos médicos tipo IIB (diretiva 93/42 CCE de 14 de junho de 1993, relativa a dispositivos médicos), destinados ao tratamento e atenuação de doenças e para fins de investigação. ${ }^{12,15}$

Através das suas propriedades fisiológicas, a OHB parece ter uma ação multifatorial com potencial benefício clínico no pé diabético: 
- Efeito anti-hipóxico: através do aumento da concentração de oxigénio dissolvido no plasma é promovida a correção da hipoxia tecidular (lei de Henry e de Dalton);

- Efeito angiogénico: desencadeia a formação de novos vasos a partir de células endoteliais locais e de vasos pré-existentes; está também descrito o recrutamento de células estaminais ou progenitoras de células circulantes através da estimulação da enzima óxido nítrico sintetase endotelial medular.

- Efeito pró-cicatrizante: estimulação da síntese de colagénio pelos fibroblastos e a sua deposição na matriz extracelular, favorecendo o processo da reepitelização;

- Efeito anti-infecioso: contribui para a atividade antimicrobiana, principalmente para microrganismos anaeróbios, através do sinergismo com alguns antibióticos, do efeito bactericida e bacteriostático e pela promoção da diapedese e fagocitose dos leucócitos; de destacar o efeito local obtido pelo aumento da concentração do antibiótico na zona da lesão;

- Efeito anti-inflamatório: redução de citocinas e prostaglandinas intervenientes no processo inflamatório;

- Efeito anti-edematoso - a vasoconstrição hiperóxica, não hipoxemiante, seletiva (efeito "Robin Hood") ao nível dos tecidos sãos, promove a atenuação do edema e redistribuição da volémia periférica a favor dos tecidos hipóxicos. ${ }^{15-17}$

Atendendo à evidência científica existente, o pé diabético foi considerado pelo European Committee for Hyperbaric Medicine (ECHM), em abril de 2016, como sendo uma das indicações clínicas para OHB com grau de recomendação II e nível de evidência B. ${ }^{18}$

\section{MATERIAL E MÉTODOS}

Realizada pesquisa bibliográfica na PubMed, CINAHL, Cochrane e Google Académico utilizando os termos "Diabetic foot" AND "Foot ulcer" AND "Hyperbaric oxygenation therapy". A seleção de artigos teve como base os seguintes critérios:

- Revisões sistemáticas, com caráter qualitativo ou quantitativo, de estudos prospetivos aleatorizados e controlados (EPAC). Podem ainda incluir estudos prospetivos não-aleatorizados (EPNA) e/ou de caso-controlo (ECC);

- Publicações com uma análise centrada em pelo menos dois dos seguintes outcomes: amputação major, ampu- tação minor, taxa e/ou tempo de cicatrização, redução da área da úlcera, saturação de oxigénio transcutânea, efeitos adversos, impacto na qualidade de vida e custo-efetividade;

- Em revisões de múltiplas terapêuticas complementares e/ou condições patológicas, foram valorizadas apenas as secções relativas à OHB no pé diabético;

- Revisões sistemáticas redigidas em língua inglesa, com data posterior a 2004 (inclusive).

Na pesquisa inicial, foram identificados trinta e dois artigos. Entre estes, seis foram selecionados ${ }^{13,14,19,20-22}$ após revisão manual dos resumos e a aplicação dos critérios definidos.

Foi também utilizada a Database of Randomised Trials in Hyperbaric Medicine (DORCTHIM) ${ }^{23}$ para a consulta dos estudos incluídos nas revisões sistemáticas. Quatro estudos ${ }^{24-27}$ utilizados como referência bibliográfica complementar nos artigos selecionados foram também abordados nesta revisão da literatura por serem considerados pertinentes para a discussão. Adicionalmente, foi consultado o Relatório Anual do Observatório Nacional da Diabetes 2015 e websites de entidades oficiais como Direção-Geral da Saúde e OMS.

\section{RESULTADOS}

Com a exceção das revisões sistemáticas descritivas de FL Game et al ${ }^{13}$ e de RM Stoekenbroek et al, ${ }^{21}$ todos os estudos referidos na Tabela 1 apresentaram os seus dados segundo uma avaliação qualitativa. Através da análise da Tabela 2 confirma-se que a OHB diminui o risco de amputação e, em especial, a amputação major, determinando um impacto significativo na cicatrização aos seis meses. Em úlceras de pé diabético com mais de 12 meses de evolução, a sua eficácia clínica, embora promissora, carece de maior robustez estatística. A saturação de oxigénio transcutânea do pé afetado foi também maior nos doentes tratados com $\mathrm{OHB}$. Em relação à redução do tamanho da úlcera não existem dados estatísticos que permitam estabelecer conclusões. A incidência de efeitos laterais da OHB foi baixa e sem significado estatístico, envolvendo maioritariamente eventos de barotrauma do ouvido.

Do conjunto de estudos prospetivos, aleatorizados e controlados (EPAC) analisados, destaca-se M Londahl et al24 pelo maior tamanho amostral e pelo benefício clínico demonstrado na cicatrização completa das úlceras ao final de 12 meses (52\% OHB versus 29\% placebo; $p=0,03$ ) e com melhoria da qualidade de vida a longo prazo. 
TABELA 1. Critérios de inclusão, exclusão e parâmetros analisados nas revisões sistemáticas selecionadas.

\begin{tabular}{|c|c|c|c|c|}
\hline $\begin{array}{l}\text { Revisão } \\
\text { sistemática }\end{array}$ & $\begin{array}{l}\mathrm{N}^{\circ} \text { e características } \\
\text { dos estudos incluídos }\end{array}$ & ND & $\begin{array}{l}\text { Comparadores e critérios de inclusão } \\
\text { e exclusão }\end{array}$ & Parâmetros analisados \\
\hline $\begin{array}{l}\text { | Roeckl- } \\
\text {-Wiedmann } \\
\text { et al, 2005'19 }\end{array}$ & $\begin{array}{l}5 \text { EPAC } \\
\text { Total: } 5 \text { estudos }\end{array}$ & 175 & $\begin{array}{l}\text { - OHB versus placebo ou OHB versus outro } \\
\text { tratamento que não a OHB; } \\
\text { - DM tipo } 1 \text { ou 2; } \\
\text { · Úlceras crónicas sem melhoria após tratamen- } \\
\text { to prévio que não incluísse OHB; } \\
\text { · Excluídos estudos com oxigénio hiperbárico } \\
\text { tópico e estudos realizados em não-humanos. }\end{array}$ & $\begin{array}{l}\text { - Cicatrização de úlcera; } \\
\text { - Redução da área da úlcera; } \\
\text { - Taxa de amputação major/minor; } \\
\text { - Variação da saturação de oxigénio } \\
\text { transcutânea; } \\
\text { - Efeitos adversos. }\end{array}$ \\
\hline $\begin{array}{l}\text { FL Game et al, } \\
2012^{13}\end{array}$ & $\begin{array}{l}2 \text { EPAC } \\
1 \text { ECC } \\
\text { Total: } 3 \text { estudos }\end{array}$ & 236 & $\begin{array}{l}\text { - OHB versus placebo ou OHB versus outro } \\
\text { tratamento que não a OHB; } \\
\text { - DM tipo } 1 \text { ou 2; } \\
\text { - Úlceras crónicas do membro inferior. }\end{array}$ & $\begin{array}{l}\text { - Cicatrização de úlcera; } \\
\text { · Tempo de cicatrização; } \\
\text { · Redução da área da úlcera; } \\
\text { · } \text { Amputação. }\end{array}$ \\
\hline $\begin{array}{l}\text { R Liu et al, } \\
2013^{20}\end{array}$ & $\begin{array}{l}7 \text { EPAC } \\
4 \text { EPNA } \\
2 \text { ECC } \\
\text { Total: } 13 \text { estudos }\end{array}$ & 624 & $\begin{array}{l}\text { - OHB versus tratamento convencional ou OHB } \\
\text { versus tratamento não convencional que não } \\
\text { a OHB; } \\
\text { - DM tipo } 1 \text { e 2; } \\
\text { - Úlceras crónicas da extremidade distal infe- } \\
\text { rior; } \\
\text { - Estudos que reportem controlo da glicemia, } \\
\text { revascularização, desbridamento, alívio da } \\
\text { carga sobre a úlcera e ainda controlo } \\
\text { metabólico e da infeção. }\end{array}$ & $\begin{array}{l}\text { - Cicatrização de úlcera a curto } \\
\text { (seguimento } \leq 6 \text { meses) e longo- } \\
\text {-prazo (seguimento } \geq 12 \text { meses); } \\
\text { - Amputação major/minor; } \\
\text { - Análise custo-eficácia; } \\
\text { - Qualidade de vida; } \\
\text { - Eventos relacionados com infeção; } \\
\text { - Efeitos adversos. }\end{array}$ \\
\hline $\begin{array}{l}\text { RM } \\
\text { Stoekenbroek } \\
\text { et al, } 2014^{21}\end{array}$ & $\begin{array}{l}7 \text { EPAC } \\
\text { Total: } 7 \text { estudos }\end{array}$ & 376 & $\begin{array}{l}\text { OHB versus tratamento convencional } \\
\text { combinado ou não com a OHB; } \\
\text { DM tipo } 1 \text { e 2; } \\
\text { - Úlceras crónicas da extremidade distal } \\
\text { inferior. }\end{array}$ & $\begin{array}{l}\text { - Cicatrização de úlcera; } \\
\text { - Redução da área da úlcera; } \\
\text { - Amputação major/minor; } \\
\text { - Necessidade de intervenções } \\
\text { terapêuticas adicionais; } \\
\text { - Efeitos adversos. }\end{array}$ \\
\hline $\begin{array}{l}\text { P Kranke et al, } \\
2015^{22}\end{array}$ & $\begin{array}{l}10 \text { EPAC } \\
\text { Total: } 10 \text { estudos }\end{array}$ & 531 & $\begin{array}{l}\text { - OHB versus tratamento que não inclua a OHB; } \\
\text { - DM tipo } 1 \text { e } 2 \text {; } \\
\text { · Úlceras crónicas do membro inferior. }\end{array}$ & $\begin{array}{l}\text { - Cicatrização de úlcera em } \\
6 \text { semanas, } 6 \text { e } 12 \text { meses; } \\
\text { - Amputação major e minor; } \\
\text { - Variação da saturação do oxigénio } \\
\text { transcutânea; } \\
\text { - Redução da área da úlcera; } \\
\text { - Qualidade de vida; } \\
\text { - Efeitos adversos. }\end{array}$ \\
\hline $\begin{array}{l}\text { T Elraiyah et al, } \\
2016^{14}\end{array}$ & $\begin{array}{l}7 \text { EPAC } \\
7 \text { EPNA } \\
2 \text { ECC } \\
\text { Total: } 16 \text { estudos }\end{array}$ & 7570 & $\begin{array}{l}\text { OHB versus placebo versus tratamento que } \\
\text { não inclua a OHB, como dispositivos de pres- } \\
\text { são arterial e agentes farmacológicos (pento- } \\
\text { xifilina, cilostazol e iloprost); } \\
\text { DM tipo } 1 \text { e 2; } \\
\text { - Úlceras crónicas do membro inferior. }\end{array}$ & $\begin{array}{l}\text { - Cicatrização completa de úlcera; } \\
\text { · Amputação major. }\end{array}$ \\
\hline
\end{tabular}

EPAC - estudos prospetivos, aleatorizados e controlados; EPNA - estudos prospetivos e não-aleatorizados; ECC - estudos de caso-controlo; ND - número de doentes com úlcera de pé diabético envolvidos; DM - diabetes mellitus; OHB - oxigenoterapia hiperbárica.

\section{DISCUSSÃO E PERSPETIVAS FUTURAS}

Em termos teóricos, a utilização da OHB no pé diabético poderá facilitar o processo de encerramento da úlcera a curto prazo, diminuir o risco da sua recorrência e a amputação major, melhorando a qualidade de vida destes doentes. Consequentemente, nos diferentes estudos, os outcomes "amputação major" e "cicatrização" são transversalmente avaliados. A amputação minor, a variação da oxigenação transcutânea, a área da úlcera e a documentação dos efeitos adversos também permitem uma avaliação do seu potencial benefício clínico. Para além do referido, alguns estudos incluem uma análise de custo-efetividade e de qualidade de vida dos doentes tratados.

O resultado da análise dos estudos, de uma forma geral, sugere um benefício da OHB. Não obstante, o caráter retrospetivo, não aleatorizado ou de estudo-caso pode condicionar a confiabilidade dos resultados apresentados. Para além disso, alguns dados dizem respeito a estudos que decorreram há mais de duas décadas, pelo que se encontram desadequados face ao atual conhecimento científico e desenvolvimento tecnológico.

Apesar do maior poder estatístico dos EPAC, os tamanhos amostrais não foram significativos. Por outro lado, a inclusão de EPNA e de ECC enviesou a análise global 
TABELA 2. Oxigenoterapia hiperbárica: síntese dos resultados apontados pelas revisões sistemáticas.

\begin{tabular}{l|l|l|l}
$\begin{array}{l}\text { Revisão } \\
\text { sistemática }\end{array}$ & $\begin{array}{l}\text { Caráter quantitativo/ } \\
\text { /qualitativo da análise }\end{array}$ & Benefício clínico documentado da OHB & $\begin{array}{l}\text { Efeitos adversos relacionados } \\
\text { com a utilização da OHB }\end{array}$ \\
$\begin{array}{lll}\text { I Roeckl- } \\
\text {-Wiedmann }\end{array}$ & Quantitativo & $\begin{array}{l}\text { Diminuição do risco de amputação major (RR=0,31); } \\
\text { et al, } 2005^{19}\end{array}$ & $\begin{array}{l}\text { Aumento da saturação de oxigénio transcutânea (WMD: } \\
11,76 \mathrm{mmHg} \text { ). }\end{array}$
\end{tabular}

\begin{tabular}{|c|c|c|c|}
\hline $\begin{array}{l}\text { FL Game et al, } \\
2012^{13}\end{array}$ & Qualitativo & $\begin{array}{l}\text { Diminuição significativa do número de amputações } \\
\text { major; } \\
\text { · Melhoria da cicatrização sem necessidade de interven- } \\
\text { ção cirúrgica; } \\
\text { · Melhoria da cicatrização aos } 12 \text { meses. }\end{array}$ & Sem referência. \\
\hline $\begin{array}{l}\text { R Liu et al., } \\
2013^{20}\end{array}$ & Quantitativo & $\begin{array}{l}\text { - } \text { Melhoria da cicatrização aos } 6 \text { meses (RR 1,50; } p=0,02) \\
\text { e para além dos } 12 \text { meses (RR 2,97; } p=0,02) \text {; } \\
\text { - Diminuição do risco de amputação major (RR 0,29; } \\
\text { - } \text { Diminuição do risco total de amputação (RR 0,29); } \\
\text { - } \text { Contradição relativa à qualidade de vida a longo-prazo }\end{array}$ & $\begin{array}{l}\text { Três estudos referem barotrauma, } \\
\text { hiperóxia, claustrofobia e efeitos } \\
\text { oculares; sem diferença significati- } \\
\text { va dos efeitos adversos entre } \mathrm{OHB} \\
\text { e controlo (RR } 1,41 ; p=0,37) \text {. }\end{array}$ \\
\hline
\end{tabular}

\begin{tabular}{|c|c|c|c|}
\hline $\begin{array}{l}\text { RM } \\
\text { Stoekenbroek } \\
\text { et al, 2014 }\end{array}$ & Qualitativo & $\begin{array}{l}\text { Potencial benefício no processo de cicatrização com } \\
\text { isquemia arterial concomitante. }\end{array}$ & $\begin{array}{l}\text { Cinco estudos referem hipoglice- } \\
\text { mia }(n=4) \text {, otite barotraumática } \\
(n=2) \text {, tonturas e agravamento de } \\
\text { cataratas }(n=1) \text {. }\end{array}$ \\
\hline $\begin{array}{l}\text { P Kranke et al, } \\
2015^{22}\end{array}$ & Quantitativo & $\begin{array}{l}\text { Melhoria da cicatrização às } 6 \text { semanas (RR 2,35; } \\
\text { p=0,01); } \\
\text { - Aumento da saturação de oxigénio transcutânea (WMD: } \\
\text { 11,8 mmHg); }\end{array}$ & $\begin{array}{l}\text { Três estudos referem claustro- } \\
\text { fobia }(n=2) \text {, barotraumatismo do } \\
\text { ouvido }(n=1) \text {, acufenos }(n=1) \text { e } \\
\text { cefaleias }(n=1) \text {. }\end{array}$ \\
\hline
\end{tabular}

- Maior redução da área da úlcera (2 EPAC);

- Melhoria da qualidade de vida a curto e longo-prazo (1 EPAC).

\begin{tabular}{l|l|l|}
\hline $\begin{array}{l}\text { Tarig Elraiyah, } \\
\text { et al, } 2016^{14}\end{array}$ & Quantitativo & Melhoria da cicatrização (OR 14,25); \\
- Diminuição do risco de amputação major (OR 0,30).
\end{tabular}

EPAC - estudo prospetivo aleatorizado e controlado; OHB - oxigenoterapia hiperbárica; RR - risco relativo; WMD - diferença média ponderada; OR - odds ratio.

dos resultados. ${ }^{13,14,20,21}$ Destaca-se, a título de exemplo, RM Stoekenbroek et al ${ }^{21}$ que previam realizar inicialmente uma meta-análise, mas que se revelou impossível pela heterogeneidade dos dados analisados. Para além das limitações inerentes a cada tipologia de estudo, há a destacar a variabilidade das metodologias consideradas, assim como o perfil clínico de cada doente tratado. Adicionalmente, o protocolo de OHB aplicado e as definições dos parâmetros "amputação major" e cicatrização de úlcera também não foram uniformizados transversalmente nos estudos analisados.

A maior parte dos estudos recorreu à utilização do sistema de Wagner para a classificação de úlceras. No entanto, este revela-se pouco objetivo e detalhado em comparação com outras escalas existentes, como é exemplo a escala de Texas. Atualmente estão também disponíveis no mercado aparelhos que permitem uma colheita digital das dimensões da úlcera que facilitam o processo de registo. ${ }^{28}$

O tempo de seguimento dos doentes incluídos é divergente entre os estudos e foi muitas vezes insuficiente para obter conclusões do tratamento a longo-prazo. Constata-se também que a etiologia das úlceras incluídas nos grupos de tratamento e de controlo é variável: certos estudos incluem úlceras isquémicas, outras úlceras não-isquémicas, havendo ainda os que não prestam qualquer esclarecimento quanto à presença de doença vascular periférica. ${ }^{21}$

Na individualidade dos EPAC analisados, o estudo de $M$. Londahl et $a^{24}$ pode ser estatisticamente questionado em dois pontos de análise. Por um lado, apenas 55\% dos doentes elegíveis foram avaliados no seguimento de um ano. Por outro, o número de amputações major foi reduzido em ambos os braços em estudo, o que torna difícil retirar ilações sobre o efeito terapêutico da OHB. ${ }^{26}$

Enumeram-se ainda três estudos considerados relevantes para a discussão, por contraporem os benefícios da OHB neste contexto.

Fedorko et al ${ }^{26}$ (107 doentes submetidos a tratamento convencional e a 30 sessões de $\mathrm{OHB}$ versus doentes sob tratamento convencional), concluiu que a OHB não conferiu, às 12 semanas, redução da indicação para amputa- 
ção (OR 0,91; $p=0,846)$, nem de facilitação do processo de cicatrização (OR 0,90; p=0,823). ${ }^{26}$ Estes dados corroboram o estudo de coorte multicêntrico e retrospetivo de Margolis et al ${ }^{18}$ (793 doentes submetidos a OHB versus 5466 sob tratamento convencional), sugerindo que não existiria benefício clínico da OHB [hazard ratio (HR) $0,68]$. Aliás, foram mesmo registadas maiores taxas de amputações major no grupo de OHB (HR 2,37) depois de 16 semanas de seguimento. ${ }^{25}$

Contudo, o estudo Fedorko $L$ et a ${ }^{26}$ pode ser criticado em várias vertentes. A avaliação do parâmetro "amputação" revela uma discrepância entre o protocolo do estudo validado e a concretização efetiva. O protocolo publicado definia o envio do doente, seis semanas após o término do tratamento, a um cirurgião vascular para avaliação da necessidade de amputação em consulta. No entanto, verifica-se que tal avaliação foi feita à distância com base em fotografias da úlcera e informação clínica. Tal procedimento não está cientificamente comprovado. Por outro lado, aos doentes tratados com OHB, foi administrado um número considerado subótimo de sessões para que possam ser garantidos resultados clinicamente significativos. ${ }^{29} \mathrm{O}$ curto seguimento de seis semanas após conclusão do tratamento é justificado pelos autores com o facto do estudo incluir apenas úlceras classificadas em grau 1 e 2 de Wagner e de excluir doentes com isquemia e infeção da ferida. No entanto, além do período de seis semanas ser considerado insuficiente para a obtenção de conclusões fidedignas, o perfil das úlceras incluídas não é considerado representativo dos doentes com pé diabético. ${ }^{29}$

Por outro lado, o ponto forte do estudo de Margolis et $a^{25}$ centra-se no elevado número de doentes tratados com OHB. Não obstante, várias limitações também podem ser apontadas. Em primeiro lugar, o inevitável viés introduzido pelo caráter retrospetivo do estudo. Em segundo, a maioria das úlceras envolvidas foram classificadas com o grau II no sistema de Wagner antes do tratamento, enquanto outros estudos prospetivos já realizados abrangeram graus de maior gravidade. Em terceiro lugar, é necessário um seguimento superior a 16 semanas para que um benefício significativo da $\mathrm{OHB}$ possa ser demonstrado. Por último, a coexistência de doença arterial periférica nos doentes tratados não é referida em momento algum. ${ }^{21}$

Recentemente, o ensaio clínico DAMO2CLES ${ }^{27}$ aleatorizou doentes diabéticos com úlcera isquémica crónica dos membros inferiores para tratamento convencional versus tratamento convencional combinado com até 40 sessões de OHB. Houve diferenças na diminuição do risco de amputação (10\%) e da sobrevivência livre de amputação (13\%) a favor do braço terapêutico com OHB. Por outro lado, a diferença no processo de cicatrização completa, para além dos 12 meses, foi apenas de $3 \%$ a favor do último. Os autores concluíram que a OHB não teria aumentado de forma significativa a cicatrização completa ou a preservação do membro inferior em doentes diabéticos com úlcera isquémica crónica dos membros inferiores.

No entanto, apesar do DAMO2CLES corresponder a um ensaio clínico aleatorizado multicêntrico e de ter uma previsão inicial de 226 doentes com úlceras isquémicas crónicas com grau Wagner 2-4 (com avaliação antecipada da indicação para revascularização), existem inúmeras vieses metodológicos que importam ser salientados e que mereceram a atenção dos especialistas na área. ${ }^{30-32}$ Existe uma discrepância significativa entre a previsão inicial do tamanho amostral $(n=226)$ e o número de doentes recrutados $(n=120)$. Neste âmbito, poderemos estar perante um erro estatístico do tipo II, uma vez que a tendência dos endpoints deste estudo são a favor do braço experimental com OHB, não tendo sido alcançada a significância estatística. Noutro âmbito, o protocolo do estudo estabelece que o ciclo de tratamentos com OHB compreenderia um número máximo de 40 sessões ou até à cicatrização completa da úlcera do membro inferior. Contudo, na análise dos resultados, o número máximo de sessões é redefinido para um máximo de 30, contrariando o estipulado no protocolo inicial do estudo. De salientar, que 30 sessões é considerado, por centros especializados, como sendo um tratamento subótimo para o contexto do pé diabético, principalmente se se incluírem casos mais gravosos. Para além do referido, a estratificação da gravidade das úlceras diabéticas incluídas nos dois braços não foi equilibrada, com $42 \%$ dos doentes com úlceras de graus III e IV no braço de controlo e 55\% no braço experimental com OHB. As úlceras de maior gravidade são mais propensas a osteomielite e, por conseguinte, à maior necessidade de tratamentos com OHB mais prolongados (para além das 30 sessões) e até ao maior risco de amputação do membro inferior. Por outro lado, apesar de se tratar de um ensaio multicêntrico ( $n=25$ ), não estão definidos o número de doentes que estão alocados a cada centro, nem qual é o protocolo do tratamento convencional em cada um deles, variáveis que também poderão influenciar na análise dos resultados. No que concerne o tratamento com a OHB, 15\% dos doentes aleatorizados para o braço experimental não chegaram a iniciar este tipo de tratamento e apenas 65\% vieram a completar as 30 sessões de OHB (por decisão dos próprios doentes, por ausência de critérios para a OHB, por necessidade de internamento hospitalar ou de outro tipo de tratamento e por 
morte). Estes dados vêm diminuir ainda mais o subgrupo de doentes tratados com OHB e suportam, mais uma vez, a importância da seleção adequada dos doentes para o tratamento com a OHB. Para finalizar, constatou-se, respetivamente, que em 56\% e 45\% das úlceras diabéticas incluídas nos braços do tratamento de controlo e experimental, o seu grau era o II de Wagner, contrariando as orientações da UHMS que não recomenda a $\mathrm{OHB}$ neste estadio.

A discussão na comunidade científica mantém-se dividida entre os diferentes estudos realizados até à data, havendo ainda questões que carecem de uma resposta mais objetiva. A taxa de recorrência da úlcera do pé diabético de cerca de 70\% num período de 5 anos determina a necessidade de estudar as intervenções terapêuticas durante um período de seguimento mais longo.33 A evidência atual neste parâmetro não é ainda suficientemente esclarecedora, segundo os resultados analisados. Conduzir um EPAC desta natureza é um processo dispendioso, especialmente quando a dimensão da amostra em causa determina estatisticamente a qualidade e universalidade dos resultados.

Demonstrou-se anteriormente que não são ainda conhecidas inequivocamente as características clínicas que devem conduzir à prescrição de $\mathrm{OHB}$. Por ser uma modalidade terapêutica dispendiosa, torna-se necessário estabelecer com rigor científico os fatores preditivos de maior resposta a este tratamento especializado. Foi neste sentido que parâmetros clínicos como a gravidade e o número das úlceras, a dependência de insulina, os valores da oximetria de pulso e a insuficiência renal foram apontados pela análise de Bishop AJ et al ${ }^{34}$ como potenciais determinantes ao ponderar o uso da OHB no algoritmo de tratamento da úlcera do pé diabético. $\bigcirc$ trabalho destes autores corroborou ainda as conclusões de Otto $G$ et $a^{35}$ ao verificar que o consumo de tabaco acima de quarenta maços-ano tem um impacto negativo importante na efetividade do tratamento com $\mathrm{OHB}$. Por outro lado, nos grupos de doentes fumadores com consumos abaixo daquele valor e de doentes que nunca fumaram a resposta clínica foi bastante favorável. Desta forma, é necessário que novos ensaios aleatorizados e prospetivos procurem validar a aplicação destes parâmetros com vista a maximizar o custo-benefício da $\mathrm{OHB}$ a curto, médio e longo prazo.

A avaliação vascular imagiológica e a saturação transcutânea de oxigénio deverão ser consideradas antes e após a realização de cada ciclo de tratamentos com OHB de forma a objetivar a sua eficácia clínica. Para além da identificação do grau de isquemia periférica, torna-se relevante documentar a existência da compo- nente neuropática ou neuroisquémica da úlcera, consubstanciando a necessidade deste tipo de tratamento complementar.

Numa perspetiva farmacoeconómica, têm de ser definidos os custos diretos e indiretos inerentes ao funcionamento e manutenção de um centro especializado em Medicina Hiperbárica. No entanto, de uma forma global e a longo prazo, o custo final de cada plano de tratamentos será, na maioria dos casos, menos dispendioso quando comparado com outras modalidades terapêuticas, incluindo a amputação major.

\section{CONCLUSÃO}

A revisão da literatura do pé diabético e, em especial, do papel da OHB neste contexto, aponta no sentido da utilidade clínica deste tipo de tratamento complementar, nomeadamente, na melhoria do processo de cicatrização e na diminuição do risco de amputação. Contudo, ainda não está definido o perfil ideal do doente com pé diabético que beneficiará da $\mathrm{OHB}$, nem o melhor protocolo de tratamento a realizar na câmara hiperbárica.

Apesar dos inúmeros estudos realizados com diferentes níveis de evidência e de qualidade metodológica, ainda persiste controvérsia em relação à eficacidade da OHB. Neste sentido, torna-se necessária a realização de mais investigação e de ensaios clínicos que respondam adequadamente à questão, ainda latente, relacionada com o benefício clínico da OHB neste contexto clínico em particular.

CONFLITOS DE INTERESSE: Os autores declaram não ter qualquer conflito de interesse na realização do presente trabalho.

FONTES DE FINANCIAMENTO: Não houve qualquer fonte de financiamento na realização do presente trabalho.

PROVENIÊNCIA E REVISÃO POR PARES: Não comissionado; revisão externa por pares.

CONFLICTS OF INTEREST: The authors declare that they have no conflicts of interest.

FINANCIAL SUPPORT: This work has not received any contribution, grant or scholarship.

PROVENANCE AND PEER REVIEW: Not commissioned; externally peer reviewed. 


\section{REFERÊNCIAS}

1. World Health Organization. Global Report on Diabetes. Geneva:WHO; 2016

2. Sociedade Portuguesa de Diabetologia. Relatório Anual do Observatório Nacional da Diabetes. Lisboa: SPD, 2015. 64p.

3. International Diabetes Federation. IDF Diabetes Atlas. 6th ed. Brussels: IDF; 2015.

4. Zhang P, Lu J, Jing Y, Tang S, Zhu D, Bi Y. Global epidemiology of diabetic foot ulceration: a systematic review and meta-analysis. Ann Med. 2016;3:1-11.

5. Associação Protetora dos Diabéticos de Portugal. Complicações [Internet]. 2016 [cited 2019 Sep 30]. Available from: http://www.apdp.pt/a-diabetes/complicacoes.

6. Santos I, Sobreira M, Nunes É, Morais M. The prevalence and factors associated with diabetic foot amputations. Ciência Saúde Coletiva. 2013;18:3007-14.

7. Assumpção E, Pitta G, Macedo A, Mendonça G, Albuquerque L, Lyra L, et al. Comparison of risk factors for major and minor amputation in diabetic patients included in a Family Health Program. J Vasc Bras. 2009;8:133-8.

8. National Institute for Health and Care Excellence: Clinical Guidelines. London: NICE; 2015.

9. Direção-Geral da Saúde. Programa Nacional de Prevenção e Controlo da Diabetes - Circular Normativa: N05/PNPCD. Lisboa: DGS; 2010.

10. Siersma V, Thorsen H, Holstein P, Kars M, Apelqvist J, Jude E, et al. Health-related quality of life predicts major amputation and death, but not healing, in people with diabetes presenting with foot ulcers: The Eurodiale Study. Diabetes Care. 2014 ;37:694-700. doi: 10.2337/dc13-1212.

11. Margolis J, Malay S, Hoffstad J, Leonard E, MaCurdy T, López $\mathrm{K}$, et al. Incidence of diabetic foot ulcer and lower extremity amputation among Medicare beneficiaries, 2006 to 2008: Data Points \#2. Data Points Publication Series. [cited 2019 Oct 3]. Available from: https://www.ncbi.n/m.nih.gov/books/ NBK65149/.

12. Albuquerque e Sousa J. Hyperbaric oxygen therapy. Historical perspective, physiological mechanisms and clinical applications. J Port Soc Intern Med. 2007;14:219-27.

13. Game FL, Hinchliffe RJ, Apelqvist J, Armstrong DG, Bakker K, Hartemann A, et al. A systematic of interventions to enhance the healing of chronic ulcers of the foot in diabetes. Diabetes Metab Res Rev. 2012;28:119-41. doi: 10.1002/dmrr.825.

14. Elraiyah T, Tsapas A, Prutsky G, Domecq J, Hasan R, Firwana B, et al. A systematic review and meta-analysis of adjunctive therapies in diabetic foot ulcers. J Vasc Surg. 2016;63:46S-58s. doi: 10.1016/j.jvs.2015.10.007.

15. Costa DA, Costa TP, Netto EC, Joaquim N, Ventura I, Pratas $A C$, et al. New perspectives on the conservative management of osteoradionecrosis of the mandible: A literature review. Head Neck. 2016;38:1708-16. doi: 10.1002/hed.24495.

16. Guerreiro F. Practical Application of Hyperbaric Oxygen Therapy. North Atlantic Treaty Organization Science and Technology Organization documents [internet]. 2015 Mar 3 [cited 2019 Oct 3]. Available from: https://www.sto.nato.int/publications.

17. Thom SR. Hyperbaric Oxygen - its mechanisms and efficacy. Plast Reconstr Surg. 2011 ;127(Suppl 1):131S-141S. doi: 10.1097/PRS.0b013e3181fbe2bf.

18. Mathieu D, Marroni A, Kot J. Tenth European Consensus Conference on Hyperbaric Medicine: preliminary report. Diving Hyperb Med. 2016;46:122-3.
19. Roeckl-Wiedmann I, Bennett M, Kranke P. Systematic review of hyperbaric oxygen in the management of chronic wounds. Br J Surg. 2005;92:24-32.

20. Liu R, Li L, Yang M, Boden G, Yang G. Systematic review if the effectiveness of hyperbaric oxygenation therapy in the management of chronic diabetic foot ulcers. Mayo Clin Proc. 2013;88:166-75.

21. Stoekenbroek RM, Santema TB, Legemate DA, Ubbink DT, van den Brink A, Koelemay MJ. Hyperbaric oxygen for the treatment of diabetic foot ulcers: a systematic review. Eur J Vasc Endovasc Surg. 2014;47:647-55. doi: 10.1016/j. ejvs.2014.03.005.

22. Kranke P, Bennett MH, Martyn-St James M, Scnabel A, Debus SE, Weibel S. Hyperbaric oxygen therapy for chronic wounds. Cochrane Database Syst Rev. 2015; 6:CD004123. doi: 10.1002/14651858.CD004123.pub4.

23. Connor D, Bennett M. The Database of Randomised Controlled Trials in Diving and Hyperbaric Medicine [internet]. Sydney. 2016 [cited 2019 Oct 2]. Available from: http://hboevidence.unsw.wikispaces.net/.

24. Londahl M, Katzman P, Nilsson A, Hammarlund C. Hyperbaric oxygen therapy facilitates healing of chronic foot ulcers in patients with diabetes. Diabetes Care. 2010;33:998-1003.

25. Margolis DJ, Gupta J, Hoffstad O, Papdopoulos M, Glick HA, Thom SR, et al. Lack of effectiveness of hyperbaric oxygen therapy for the treatment of diabetic foot ulcer and the prevention of amputation: a cohort study. Diabetes Care. 2013;36:196166. doi: $10.2337 / \mathrm{dc} 12-2160$.

26. Fedorko L, Bowen JM, Jones W, Oreopoulos G, Goeree R, Hopkins RB, et al. Hyperbaric oxygen therapy does not reduce indications for amputation in patients with diabetes with nonhealing ulcers of lower limb: a prospective, double-blind, randomized controlled clinical trial. Diabetes Care. 2016;39:36299. doi: 10.2337/dc15-2001.

27. Santema B, Stoekenbroek M, Koelemay J, Reekers A, van Dortmont C, Oomen A, et al. Hyperbaric Oxygen Therapy in the Treatment of Ischemic Lower Extremity Ulcers in Patients with Diabetes: Results of the DAMO ${ }_{2}$ CLES Multicenter Randomized Clinical Trial. J Diabetes. 2018;41:e62-3. doi: 10.2337/dci17-0059.

28. Stoekenbroek M, Santema B, Koelemay J, van Hulst A, Legemate A, Reekers A, et al. Is additional hyperbaric oxygen therapy cost-effective for treating ischemic diabetic ulcers? Study protocol for the Dutch DAMOCLES multicenter randomized clinical trial? J Diabetes. 2015;7:125-32. doi: 10.1111/17530407.12155.

29. Mutluoglu M, Uzun G, Bennett M, Germonpré P, Smart D, Mathieu D. Poorly designed research does not help clarify the role of hyperbaric oxygen in the treatment of chronic diabetic foot ulcers. Diving Hyperb Med. 2016;46:133-4.

30. Mutluoglu M. Comment on Santema et al. Hyperbaric Oxygen Therapy in the Treatment of Ischemic Lower-Extremity Ulcers in Patients with Diabetes: Results of the DAMO ${ }_{2}$ CLES Multicenter Randomized Clinical Trial. J Diabetes. 2018;41:11219. doi: 10.2337/dc17-2303.

31. Huang E. Comment on Santema et al. Hyperbaric Oxygen Therapy in the Treatment of Ischemic Lower-Extremity Ulcers in Patients with Diabetes: Results of the DAMO ${ }_{2}$ CLES Multicenter Randomized Clinical Trial. J Diabetes. 2018;41:112-9. doi: 10.2337/dc17-2440.

32. Santema B, Stoekenbroek M, Koelemay J, Reekers A, van Dortmont C, Oomen A, et al. Response to Comments on Santema et al. Hyperbaric Oxygen Therapy in the Treatment of Ischemic Lower-Extremity Ulcers in Patients with Diabetes: Results 
of the DAMO ${ }_{2}$ CLES Multicenter Randomized Clinical Trial. J Diabetes. 2018;41:112-9. doi: 10.2337/dc17-0654.

33. Johnston B.R, Ha A.Y, Brea B, Liu P.Y. The mechanism of hyperbaric oxygen therapy in the treatment of chronic wounds and diabetic foot ulcer. R I Med J. 2016;99:24-7.

34. Bishop AJ, Mudge E. Diabetic foot ulcers treated with hyperbaric oxygen therapy: a review of the literature. Int Wound J. 2014;11:28-34. doi: 10.1111/j.1742-481X.2012.01034.x.

35. Otto GH, Buyukcakir C, Fife CE. Effects of smoking on cost and duration of hyperbaric oxygen therapy for diabetic patients with non-healing wounds. Undersea Hyperb Med. 2000 Summer;27(2):83-9. 